\title{
Effects of Ascorbic Acid in Alkaptonuria: Alterations in Benzoquinone Acetic Acid and an Ontogenic Effect in Infancy
}

\author{
JON A. WOLFF, BRUCE BARSHOP, WILLIAM L. NYHAN, JACK LESLIE, J. E. SEEGMILLER, \\ HARRY GRUBER, MICHAEL GARST, SUSAN WINTER, KIMBERLEE MICHALS, AND \\ REUBEN MATALON \\ Department of Pediatrics and the Institute for Molecular Genetics [J.A.W., B.B., W.L.N., J.L.], and the \\ Department of Medicine [J.E.S., H.G.], University of California San Diego, La Jolla, California; Allergan Inc. \\ [M.G.], Irvine, California; Valley Children's Hospital [S.W.], Fresno, California; and the Department of \\ Pediatrics [K.M., R.M.], University of Illinois, Chicago, Illinois
}

\begin{abstract}
The effects of ascorbic acid on the excretion of homogentisic acid and its derivative benzoquinone acetic acid were studied in two adults and three infants. The administration of relatively large amounts of ascorbic acid to the adults was followed by a disappearance of benzoquinone acetic acid from the urine, whereas the level of excretion of homogentisic acid did not change. This could have relevance to the pathogenesis of ochronotic arthritis. In the 4-mo-old infant and the 5-mo-old infant ascorbic acid in the urine may have doubled the amount of homogentisic acid, presumably through an effect on the immature $p$-hydroxyphenylpyruvic acid oxidase. Dietary reduction of the intake of tyrosine and phenylalanine substantially reduced the excretion of homogentisic acid. (Pediatr Res 26: 140-144, 1989)
\end{abstract}

Alkaptonuria is a rare inborn error of tyrosine metabolism in which deficient activity of homogentisic acid oxidase (Fig. 1) produces an accumulation of homogentisic acid and leads to debilitating ochronotic arthritis in adulthood (1,2). In 1940 Sealock et al. (3), studied the use of ascorbic acid for the treatment of a patient with alkaptonuria. Vitamin $C$ therapy did not reduce the amount of homogentisic acid excreted in the urine. It was reported to delay the darkening of the urine, presumably by preventing the oxidation of homogentisic acid. In rats with experimental alkaptonuria, vitamin $\mathrm{C}$ reduced the binding of $\left[{ }^{14} \mathrm{C}\right]$ homogentisic acid to connective tissues (4). In addition ascorbic acid was found to prevent the inhibition of the growth of cultured human articular chondrocytes in vitro by homogentisic acid (Angeles A, Finley KD, Seegmiller JE, unpublished results).

Homogentisic acid is thought to cause ochronotic arthritis following its oxidation to the labile benzoquinone acetic acid which then either binds directly, or after formation of a polymer, to biologic components such as collagen (5). Homogentisic acid polyphenol oxidase can catalyze this conversion via a semiqui-

Received June 2, 1988; accepted April 21, 1989.

Correspondence Dr. William L. Nyhan, Department of Pediatrics, M-009A, University of California San Diego, La Jolla, CA 92093-0609.

Supported by Public Health Service research Grants HD04608 and Physician Scientist Award 1K11 HD00669-01MRDD (M.A.W.), National Institute of Child Health and Human Development; Grant RR00827 from the General Clinica Research Centers Program; PHS-DK 13622; PHS NS 2150; PHS-DK 07318, National Institutes of Health, Bethesda, MD; Stallone Foundation for Autism Research, Washington, D.C.; Purine 24 Foundation, Washington, D.C., W. M Keck Foundation, Los Angeles, CA; Arthritis Foundation, Atlanta, GA; and the Clayton Foundation for Research, (Calif. Division), Houston, TX. none intermediate (5). This study was undertaken to determine whether treatment with ascorbic acid was effective in reducing the concentrations in body fluids of benzoquinone acetic acid, the putative toxic metabolite. Observations were made on three children and two adults.

\section{CASE REPORTS}

Patient 1. CM was a full-term Caucasian male baby who was born without any complications and was noted to have pink staining in his wet disposable diapers at $5 \mathrm{~d}$ of age. The diagnosis of alkaptonuria was made at $3 \mathrm{wk}$ of age when his urine was found to contain a large quantity of homogentisic acid. Otherwise, he was growing and developing well, and was without any clinical symptoms. He was first studied in the UCSD Clinical Research Center, San Diego, CA, at 5 mo of age. His wt was 6.7 $\mathrm{kg}$ at $5 \mathrm{mo}$ of age, and $12.1 \mathrm{~kg}$ at $15 \mathrm{mo}$ of age.

Patient 2. DF was a 52-y-old Caucasian man who was in good health until $39 \mathrm{y}$ of age when he developed pain in the right knee and swelling that was unresponsive to repeated aspirations of synovial fluid and antiflammatory medications. A diagnosis of alkaptonuria was made at $40 \mathrm{y}$ of age when cartilage from the right knee was observed to be ochronotic. His urine was observed to darken upon standing exposed to air. At $44 \mathrm{y}$ of age he ruptured his left Achilles tendon. At $51 \mathrm{y}$ of age he had a partial left hip replacement. He had recently developed severe chronic back pain. He had black punctate pigmentation of the external ears, tympanic membranes, face, hands, and right sclera. His wt was $68.5 \mathrm{~kg}$

Patient 3. RB was a 67-y-old Caucasian man who was first diagnosed as having alkaptonuria at $18 \mathrm{y}$ of age when his urine was found to have a positive test for reducing substance in the absence of a positive test for glucose. Homogentisic acid was found in his urine to account for the positive test for reducing substance. He was asymptomatic until 34 y of age when he developed lower back pain. Later, he developed pain in the neck for which a laminectomy was performed on the upper cervical vertebrae. At the age of 45 y he experienced stiffness of both knees. At the time of study he could not straighten his back or his knees. He had a myocardial infarction at the age of $54 \mathrm{y}$. He had had hypertension beginning at the age of $54 \mathrm{y}$ and emphysema that started at $64 \mathrm{y}$. He had smoked 11/2 packs of cigarettes per day for years. At the age of $64 \mathrm{y}$ he had a cholescystectomy. His knees were swollen bilaterally. He had black pigmentation over his face and external ears. His wt was $70.5 \mathrm{~kg}$.

Patient 4. JS was a Caucasian baby who was born full-term without any complications. He was referred to the University of Illinois Genetics and Metabolic Clinic at the age of 3 mo because 


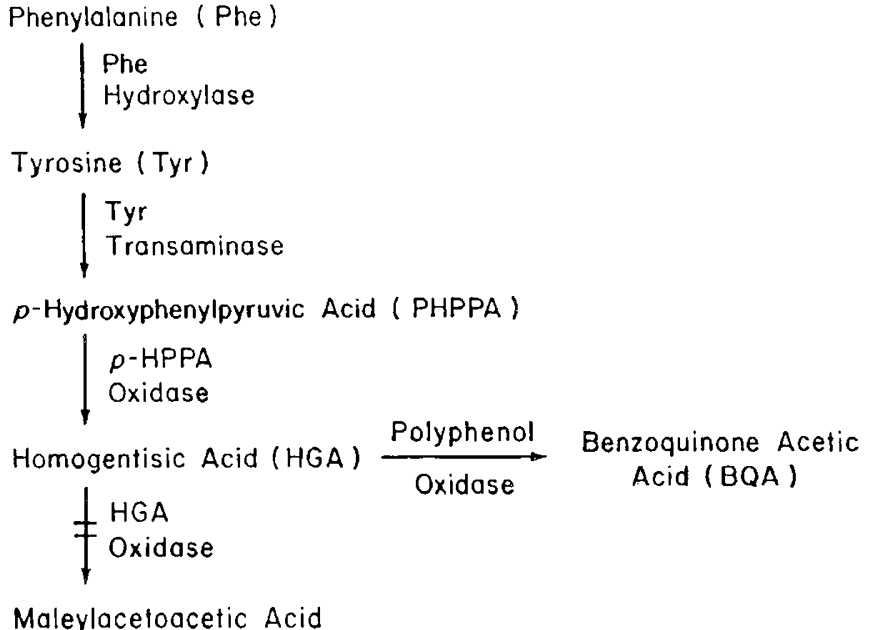

Fig. 1. Metabolic pathways relevant to alkaptonuria.

of darkening in the urine. The diagnosis of alkaptonuria was made by the finding of homogentisic acid in the urine. He entered the study at $4 \mathrm{mo}$ of age, when his height was $69 \mathrm{~cm}$ and his wt was $7.6 \mathrm{~kg}$ (both 50 th percentile for age). Vitamin C, $100 \mathrm{mg} /$ $\mathrm{kg}$, was started and daily random urine samples were collected. After 2 wk a low phenylalanine and tyrosine diet was begun.

Patient 5. SB was a full-term newborn male baby found to have pink staining of his diapers since the first days of life. The diagnosis of alkaptonuria was made at Valley Children's Hospital, Fresno, CA, at 3 mo of age, based upon measurement of large quantities of homogentisic acid in a urine sample. He exhibited normal growth and development and no evidence of pigmentation changes. Quantitation of urinary homogentisic acid was performed on samples collected at $6 \mathrm{mo}$ of age, before and after receiving $100 \mathrm{mg} / \mathrm{kg} /$ day ascorbic acid.

\section{MATERIALS AND METHODS}

Three patients were studied in the Clinical Research Center of the UCSD Medical Center. Some 2 wk before admission they were instructed to institute a diet containing no more than the recommended daily allowances of vitamin C. After admission two consecutive 24-h urine collections and two morning blood samples were obtained for the determination of levels of ascorbic acid, homogentisic acid, benzoquinone acetic acid, the semiquinone of homogentisic acid, amino acids, $p$-hydroxyphenylpyruvic acid, and $p$-hydroxyphenyllactic acid. Stools were also collected for $48 \mathrm{~h}$ and the urine and stool total nitrogen were determined and nitrogen balances were calculated. Synovial fluid was aspirated from the knees of the two adults for homogentisic acid and benzoquinone acetic acid levels. After the collections, CM was treated with $200 \mathrm{mg} / \mathrm{kg}$ of ascorbic acid per day, and the two adults were treated with $10 \mathrm{~g} /$ day of ascorbic acid, each in four divided doses. After 2 wk two 24-h urine collections and two morning blood samples were again obtained and the same studies repeated.

In $\mathrm{CM}$ the effects were studied of a diet low in phenylalanine and tyrosine, but previously shown $(6,7)$ to be sufficient for normal growth and development. During this period he received solid baby foods and a formula comprised of mixtures of Similac, Mead-Johnson (Evansville, IN) product $3200 \mathrm{AB}$, and polycose to supply a total of $0.20 \mathrm{mmol} / \mathrm{kg} /$ day of phenylalanine and tyrosine. This diet contained enough Similac and solid foods to supply $2.3 \mathrm{~g} / \mathrm{kg} /$ day of whole protein. During this period CM received only the recommended daily allowances for ascorbic acid. The studies were approved by the Human Subjects Committee of the University of California San Diego, and informed consent was obtained from each of the adult patients and from the parents of each of the children.
Assays. Urine samples were stored at $4^{\circ} \mathrm{C}$ during the 24-h collection period. The $\mathrm{pH}$ of each urine sample was adjusted to less than 5 with glacial acetic acid. If a sediment was present, the urine was warmed to room temperature to redissolve the sediment before dividing the 24-h samples into separate aliquots. Aliquots of urine for the determination of levels of phenylalanine, tyrosine, and creatinine were frozen until analysis. Aliquots of urine for homogentisic acid, benzoquinone acetic acid, the semiquinone of homogentisic acid, and ascorbic acid were mixed with an equal volume of cold column buffer concentrated $4 \times$. Column buffer was composed of 0.1 molar sodium acetate, 0.165 $\mathrm{mL} / \mathrm{L} \mathrm{N}$-octylamine and $200 \mathrm{mg}$ of sodium EDTA/L. The $\mathrm{pH}$ of the column buffer was adjusted to 4 with acetic acid. After the urine samples were thoroughly mixed with the column buffer, they were centrifuged at $2500 \mathrm{rpm}$ for $5 \mathrm{~min}$. The supernatant fluid was then kept at $-20^{\circ} \mathrm{C}$ until analysis.

Blood was removed into a heparinized syringe and immediately centrifuged. Plasma was frozen for phenylalanine and tyrosine and also mixed with an equal volume of the above column buffer and processed as the urine was above. Synovial fluid was also processed in a similar manner.

Amino acids, including phenylalanine and tyrosine, were analyzed on an automatic amino acid analyzer (8). Urinary $p$ hydroxyphenylacetate and $p$-hydroxyphenylpyruvate were measured as previously described (9).

Blood, urine, and synovial fluid were analyzed for homogentisic acid, ascorbic acid, benzoquinone acetic acid, and the semiquinone of homogentisic acid. An aliquot of each was thawed and mixed with an equal vol of a mixture containing $97 \%$ acetonitrile and $3 \%$ ethanol. The mixture was mixed and microfuged for $1 \mathrm{~min}$. The supernatant was then applied to a $\mathrm{C} 18$ microbonapack column equilibrated with column buffer. The column buffer was degassed by bubbling helium through it to remove oxygen. The flow rate was $1.5 \mathrm{~mL} / \mathrm{min}$. The sample was eluted isocratically and the effluent monitored in-line for UV absorption at $280 \mathrm{~nm}$, followed by electrochemical detection using a Bioanalytical Systems, Inc., instrument (amperometric detector LC-4, W. Lafayette, IN). The identities and calibration of the peaks were confirmed by the analysis of known standard compounds. Under oxidized conditions ascorbic acid, homogentisic acid, the semiquinone of homogentisic acid, and benzoquinone acetic acid could be detected. The semiquinone and benzoquinone acetic acids were made initially by bringing the $\mathrm{pH}$ of a solution of homogentisic acid to 7.4 in the presence of oxygen in room air. This creates a moderate conversion of homogentisic acid to the more oxidized forms. When the $\mathrm{pH}$ was raised to 9 , polymers of homogentisic acid appeared that had longer retention times on the column. The setting of the electrochemical detector for the oxidation mode was $0.6 \mathrm{~V}, 2$ $\mathrm{nAmp} / \mathrm{V}$. For the reduced mode the equipment was set at -0.2 $\mathrm{V}, 10 \mathrm{nAmp} / \mathrm{V}$. The positions of the peaks were confirmed by chromatography of benzoquinone acetic acid which had been synthesized chemically. The semiquinone was isolated and converted to the benzoquinone by alkalinization of the sample. It could be converted to the homogentisic acid peak by incubation with ascorbic acid at $37^{\circ} \mathrm{C}$.

The synthesis of 1,4-benzoquinone acetic acid was done by modification of the procedure of Fieser (10). A stirred solution of $0.04 \mathrm{~g}\left(2.38 \times 10^{-4} \mathrm{~mol}\right)$ of 2,5-dihydroxyphenylacetic acid (Sigma Chemical Co., St. Louis, MO) and $1 \mathrm{~mL}$ anhydrous tetrahydrofuran was treated in a foil-wrapped vessel with $0.11 \mathrm{~g}$ $\left(5.76 \times 20^{-4} \mathrm{~mol}\right)$ of silver oxide (Alpha-Ventron) $(11)$. After 30 min this suspension was filtered twice through glass wool. The solution was concentrated at reduced pressure. The residue was redissolved in $0.5 \mathrm{~mL}$ of anhydrous tetrahydrofuran, filtered and concentrated to leave $0.035 \mathrm{~g}$ (89\% yield) of 1,4-benzoquinone acetic acid mp $128-132^{\circ} \mathrm{C}(12)$, as a dark solid that decomposed rapidly when dissolved in acetone or tetrahydrofuran.

Analysis for urinary homogentisic acid in patient 4 was performed by gas chromatography-mass spectrometry. The urine 
was extracted with ethyl acetate and diethyl ether, and derivatized according to the method of Goodman and Markey (13). The analysis was performed on a Hewlett Packard (Palo Alto, CA) gas chromatography-mass spectrometer that used an SC 54 capillary column $25 \mathrm{M}$ long.

\section{RESULTS}

Ascorbic acid. Urinary and plasma concentrations of ascorbic acid obtained before ascorbic acid supplementation documented

Table 1. Concentrations of ascorbic acid in plasma and urine before and after oral supplementation with ascorbic acid*

\begin{tabular}{|c|c|c|c|c|}
\hline \multirow[b]{2}{*}{ Patient } & \multicolumn{2}{|c|}{$\begin{array}{l}\text { Plasma } \\
(\mathrm{mM})\end{array}$} & \multicolumn{2}{|c|}{$\begin{array}{c}\text { Urine } \\
(\mathrm{mmol} / 24 \mathrm{~h})\end{array}$} \\
\hline & - & + & - & + \\
\hline $\mathrm{CM}(5 \mathrm{mo})$ & & & $<0.001$ & 1.2 \\
\hline $\mathrm{CM}(15 \mathrm{mo})$ & 0.51 & 2.0 & 3.7 & 24.0 \\
\hline $\mathrm{DF}$ & & 1.4 & 21.6 & 97.2 \\
\hline RB & & 1.4 & 27.3 & 85.2 \\
\hline
\end{tabular}

* - , Indicates no ascorbic acid supplementation. + , indicates ascorbic acid supplementation. All values are the means of two to three separate samples each assayed three times.

Table 2. Concentrations of homogentisic acid (HGA) and benzoquinone acetic acid (BQA) in plasma and urine before and after supplementation with ascorbic acid*

\begin{tabular}{|c|c|c|c|c|c|c|}
\hline \multirow[b]{2}{*}{ Patient } & \multicolumn{2}{|c|}{$\begin{array}{l}\text { Urinary HGA } \\
(\mathrm{mmol} / 24 \mathrm{~h})\end{array}$} & \multicolumn{2}{|c|}{$\begin{array}{c}\text { Plasma HGA } \\
(\mathrm{mM})\end{array}$} & \multicolumn{2}{|c|}{$\begin{array}{l}\text { Urinary BQA } \\
(\mathrm{nmol} / 24 \mathrm{~h})\end{array}$} \\
\hline & - & + & - & + & - & + \\
\hline $\mathrm{CM}(5 \mathrm{mo})$ & 3.97 & 8.03 & NA & NA & 0.6 & $<0.1$ \\
\hline $\mathrm{CM}(15 \mathrm{mo})$ & 11.50 & 9.95 & 0.065 & 0.058 & $<0.1$ & $<0.1$ \\
\hline $\mathrm{SB}(6 \mathrm{mo})$ & 4.33 & 3.76 & NA & NA & NA & NA \\
\hline DF & 15.0 & 17.8 & 0.036 & 0.042 & 128 & $<0.1$ \\
\hline $\mathrm{RB}$ & 21.8 & 21.2 & 0.054 & 0.071 & 140 & $<0.1$ \\
\hline
\end{tabular}

* Abbreviations and units are as in Table 1. All values were the means of three separate urine collections and two or three plasmas assayed three times. NA indicates not assayed. that patients were not receiving substantial amounts of ascorbic acid before study (Table 1). Levels of ascorbic acid obtained after supplementation documented compliance with the dietary intervention. Ascorbic acid supplementation substantially increased the urinary levels of ascorbic acid (Table 1). In CM at 15 mo of age, supplementation with ascorbic acid increased the concentration of ascorbic acid in the plasma 4-fold. In patients DF and $\mathrm{RB}$, the plasma levels of ascorbic acid were considerably greater when they were receiving supplementation than the normal plasma levels of ascorbic acid (14).

Homogentisic acid. Supplementation with ascorbic acid in $\mathrm{CM}$ at 5 mo of age increased the urinary excretion of homogentisic acid to a value double that of his baseline level (Table 2). In contrast, when he was 15 mo of age, and in both adults, the urinary excretion of homogentisic acid was unaffected by ascorbic acid supplementation. Data on plasma concentrations of homogentisic acid obtained at the 15 mo time of study revealed no effect of ascorbic acid supplementation. A similar study was carried out on JS when he was 4 mo old. The data on concentrations of homogentisic acid in urine are shown in Table 3. Ascorbic acid administration increased the concentrations of homogentisic acid up to seven times $(33.04 \mathrm{mmol} / \mathrm{L})$ the control level of $4.76 \mathrm{mmol} / \mathrm{L}$. The mean of 10 samples in relation to creatinine excretion was distinctly higher. Dietary reduction of the intake of phenylalanine and tyrosine reduced these levels.

In the adult patients DF and RB synovial fluid obtained while they were not receiving ascorbic acid supplementation had concentrations of homogentisic acid of 0.037 and $0.057 \mathrm{mmol} / \mathrm{L}$, respectively. These values were remarkably similar to their respective plasma concentrations (Table 2).

Benzoquinone acetic acid. The total urinary excretion of benzoquinone acetic acid in $24 \mathrm{~h}$ for each of the patients is shown in Table 2 Before supplementation with ascorbic acid, the urinary excretion of benzoquinone acetic acid was $1.87 \mathrm{nmol} / \mathrm{kg} / 24 \mathrm{~h}$ in DF and $1.99 \mathrm{nmol} / \mathrm{kg} / 24 \mathrm{~h}$ in RB. The total urinary excretion of benzoquinone acetic acid decreased substantially during supplementation with ascorbic acid. In fact benzoquinone acetic acid was undetectable in the urine when the patients received ascorbic acid supplementation. In CM at 15 mo of age, benzoquinone acetic acid was less than the limit of detection $(<0.1)$ before supplementation.

Benzoquinone acetic acid was not detectable $(<0.1)$ in the plasma of any of the patients in the presence or absence of

Table 3. Concentrations of homogentisic acid in urine of JS in response to ascorbic acid supplementation

\begin{tabular}{lcc}
\hline & No. of samples & $\begin{array}{c}\text { Homogentisic acid } \\
\text { (mol/mol creatinine) }\end{array}$ \\
\hline Baseline 1 & 1 & 3.45 \\
2 & 1 & 3.75 \\
Ascorbic acid $(10 \mathrm{mg} / \mathrm{kg})$ & 10 & $5.59 \pm 2.36^{*}$ \\
Ascorbic acid and low phe/tyr diet & 6 & $2.27 \pm 1.25^{*}$ \\
\hline
\end{tabular}

$* \pm 1 \mathrm{SD}$

Table 4. Detailed analysis of intermediates of phenylalanine metabolism in CM at 5 mo of age*

\begin{tabular}{|c|c|c|c|c|c|c|c|}
\hline \multicolumn{2}{|c|}{ Diet } & \multicolumn{5}{|c|}{$\begin{array}{l}\text { Urinary output } \\
(\mathrm{mmol} / 24 \mathrm{~h})\end{array}$} & \multirow[b]{2}{*}{$\begin{array}{c}\text { Out/in } \\
(\%)\end{array}$} \\
\hline $\mathrm{AA}$ & Phe + Tyr & $\begin{array}{c}\text { Phe } \\
+ \\
\text { Tyr }\end{array}$ & HGA & SQ & BQA & Total output & \\
\hline 0.341 & 8.43 & 0.067 & 3.97 & 0.018 & 0.0006 & 4.15 & 47.9 \\
\hline 7.655 & 8.43 & 0.138 & 8.93 & $<0.0001$ & $<0.0001$ & 8.15 & 96.9 \\
\hline 0.341 & 2.19 & 0.03 & 0.44 & 0.0031 & 0.0006 & 0.47 & 21.5 \\
\hline
\end{tabular}

* Under the heading Diet, AA indicates the intake of ascorbic acid in mmol/24 $\mathrm{h}$ and Phe + Tyr indicates the intake of phenylalanine and tyrosine in mmol/24 h. Under the heading Urinary Output, HGA indicates homogentisic acid, SQ indicates semiquinone of homogentisic acid, BQA indicates benzoquinone acetic acid; and total output indicates these amounts added together. \% Out/in was calculated by dividing the value for total output by the dietary intake of phenylalanine and tyrosine and multiplying by 100 . All measurements represent the means of two or three separate collections each assayed three times, except for the phenylalanine and tyrosine that were quantified once. 
ascorbic acid supplementation. Similarly, benzoquinone acetic acid was not detected in synovial fluid.

Intake and output of tyrosyl compounds. The urinary output of tyrosine metabolites at 5-6 mo of age in CM was compared with the intake of phenylalanine and tyrosine (Table 4). The effects of ascorbic acid supplementation and of dietary reduction of phenylalanine and tyrosine are indicated. Total urinary output of tyrosine metabolites was determined by adding the outputs of phenylalanine, tyrosine, homogentisic acid, semiquinone of homogentisic acid, and benzoquinone acetic acid. p-Hydroxyphenyllactate and $p$-hydroxyphenylacetate were not detected in the urine of any of the patients. Total intakes of phenylalanine and tyrosine were computed from the dietary intake of protein while in the Clinical Research Center. At this age ascorbic acid increased the excretion of tyrosine metabolites from 48 to $97 \%$ of total intake of phenylalanine and tyrosine. Not only was the excretion of homogentisic acid increased, but that of the amino acids was doubled. The nitrogen balance of CM was not affected by ascorbic acid during this time.

Dietary restriction of the intake of phenylalanine and tyrosine led to the reduction in the amounts of homogentisic acid. The urinary content approximated one-tenth that of the control diet. Nevertheless, there were still appreciable amounts of benzoquinone acetic acid and the semiquinone in the urine. $\mathrm{CH}$ was in positive nitrogen balance and continued to grow at a normal rate while on this restricted diet.

\section{DISCUSSION}

The major morbidity in patients with alkaptonuria is the damage to connective tissue of joints which leads to the debilitating ochronotic arthritis. The hypotheses with which these studies were undertaken were that this was a consequence of oxidation of the homogentisic acid that accumulates in alkaptonuria to benzoquinone acetic acid (5) and that the effects of this reactive intermediate on tissues could be prevented by inhibiting the conversion of homogentisic acid to benzoquinone acetic acid by treatment with large doses of ascorbic acid (Fig. 1). To pursue this study, methodology was developed to assess the levels of benzoquinone acetic acid in body fluids. The method is convenient in that each assay provides quantification of homogentisic acid, the semiquinone of homogentisic acid, and ascorbic acid as well, in the chromatography of each sample. Ascorbic acid supplementation was found to reduce significantly the levels of benzoquinone acetic acid in the urine. In fact neither this compound nor the semiquinone were detectable during treatment with ascorbic acid. Benzoquinone acetic acid was not detectable in the plasma or synovial fluid before treatment. Concentrations of homogentisic acid in plasma were also quite low by comparison with amounts in the urine and, in two adults, the levels found in joint fluid were nearly identical to those of plasma. It was of interest and potentially useful for future studies that the plasma so closely mirrored the concentration of homogentisic acid in joint fluid. However, the very low levels in both indicate that homogentisic acid does not accumulate, but is rather bound to tissues or effectively excreted in the urine. Presumably this was also true of benzoquinone acetic acid, but it appears likely that as benzoquinone acetic acid is formed it is quickly bound to connective tissue. Data have been published by Zannoni et al. (5), indicating that binding to tissue is very rapid in vitro, and the administration of ${ }^{14} \mathrm{C}$-homogentisic acid to rats was followed by very rapid binding of isotope to xiphoid cartilage and tail tendon (4). The observed effect of ascorbic acid could represent reduction in the conversion of homogentisic acid to benzoquinone acetic acid throughout the body. It should also be recognized that the presence of large amounts of ascorbic acid in the urine might promote the conversion of benzoquinone acetic acid to homogentisic acid after it has been excreted into the urine. This is an important issue that must be resolved with further studies. Observation, and ideally quantitation, of ochronotic pigment and documentation of its change over time would be one approach. Detection of the quinone derivatives in plasma would require methods more sensitive than currently available.

The ability of ascorbic acid to cause the disappearance of benzoquinone acetic acid suggests its possible use in the prevention of ochronosis in patients with alkaptonuria. Definitive proof of efficacy will require a long-term study of a number of patients with alkaptonuria, ideally treated before the onset of symptoms of ochronosis. Variability in the times of development as well as the severity of clinical manifestations of ochronosis are such that a double-blind controlled study might be required.

Dietary restriction of the intake of phenylalanine and tyrosine led to a major reduction in the amounts of homogentisic acid in the urine. The reduction was by a factor approximating 10 , and the total excretion of phenylalanine and its products decreased to $22 \%$ of their intake, indicating that much of what was ingested was being used for anabolism. It does not appear likely that longterm compliance with extreme reduction of intake would be possible or that such an approach would be desirable in view of the prolonged time between the accumulation of homogentisic acid and the development of clinical manifestations. Nevertheless, a combination of a modest reduction in the intake of precursors of homogentisic acid along with the administration of ascorbic acid might be prudent and feasible.

Supplementation with ascorbic acid did not affect the levels of homogentisic acid in urine or plasma of the two adults studied and of the infant at a maturity level of 15 mo of age. These observations were consistent with the results of previous studies $(3,15,17)$. The response in the 5 -mo-old was very different. His excretion of homogentisic acid doubled in response to the administration of ascorbic acid. Excretion of phenylalanine and tyrosine also increased, and the total excretion of phenylalanine and its metabolic products rose to approximate $97 \%$ of the dietary intake, but the amounts of the amino acids excreted were so small that $99 \%$ of these total metabolic products was made up by homogentisic acid. A similar increase in urinary levels of homogentisic acid was also observed in JS at 4 mo. The increase in homogentisic acid excretion in these infants with alkaptonuria in our view most likely reflects an ontogenic aspect of $p$-hydroxyphenylpyruvic acid oxidase. The late development of this enzyme is thought to be the basis of the transient tyrosinemia of the newborn that is particularly common in premature infants. Ascorbic acid is effective in decreasing the levels of tyrosine in such infants (16). The increase in homogentisic acid was interpreted by us to represent a similar activation of $p$-hydroxyphenylpyruvic acid oxidase in vivo in young infants. We recognize that this is speculative, but it should be testable by studies in young animals in which there should be greater conversion of labeled tyrosine to carbon dioxide if this pathway were activated.

\section{REFERENCES}

1. Bunim JJ, McGuire JS, Hilbish TF, Laster L, La Du BN, Seegmiller JE 1957 Alcaptonuria: Clinical Staff Conference at the National Institutes of Health. Ann Intern Med 47:1210-1224

2. La Du BN, Zannoni VG, Laster L, Seegmiller JE 1958 The nature of the defect in tyrosine metabolism in alcaptonuria. J Biol Chem 230:251-260

3. Sealock RR, Galdston M, Steele JM 1940 Administration of ascorbic acid to an alkaptonuric patient. Proc Soc Exp Biol Med 44:580-583

4. Lustberg TJ, Schulman JD, Seegmiller JE 1970 Decreased binding of ${ }^{14} \mathrm{C}$ homogentisic acid induced by ascorbic acid in connective tissue of rats with experimental alcaptonuria. Nature 228:770-771

5. Zannoni VG, Lomtevas N, Goldfinger S 1969 Oxidation of homogentisic acid to ochronotic pigment in connective tissue. Biochim Biophys Acta 177:94105

6. Ney DN, Bay C, Saudubray J-M, Kelts DG, Kulovich S, Sweetman L, Nyhan WL 1985 An evaluation of protein requirements in methylmalonic acidaemia. J Inherited Metab Dis 8:132-142

7. Ney D, Bay C, Schneider JA, Kelts D, Nyhan WL 1983 Dietary management of oculocutaneous tyrosinemia in an 11-year-old child. Am J Dis Child 137:995-1000

8. Spackman DH, Stein WH, Moore S 1958 Automatic recording apparatus for use in the chromatography of amino acids. Anal Chem 30:1 190-1206

9. Sweetman L 1984 Qualitative and quantitative analysis of organic acids in physiologic fluids for diagnosis of the organic acidurias. In: Nyhan WL (ed) 
Abnormalities in Amino Acid Metabolism in Clinical Medicine. AppletonCentury-Crofts, E. Norwalk, CT, pp. 419-453

10. Feiser LF 1939 Synthesis of vitamin K. J Am Chem Soc 61:3467-3475

11. Seyforth D, Spohn RJ 1969 The reaction of organomercuric halides with dicobalt octacarbonyl. A new ketone synthesis. J Am Chem Soc 91:30373044

12. Morner CT 1912 Weitere Beiträge zur Chemie der Homogentisinsäure. Z Physiol Chem 78:307-329

13. Goodman S, Markey SP 1981 Diagnosis of organic acidemias by gas chromatography. In: Goodman S, Markey SP (eds) Laboratory and Research Meth- ods in Biology and Medicine. Alan R Liss, New York, pp. 1-43

14. Sauberlich HE 1975 Vitamin C status: methods and findings. Ann NY Acad Sci 258:438-450

15. Neuberger A, Rimington C, Wilson JM 1947 Studies on alcaptonuria. II. Investigations on a case of human alcaptonuria. Biochem J 41:438-449

16. Levine SZ, Gordon HH, Marples EJ 1941 A defect in the metabolism of tyrosine and phenylalanine in premature infants. II. Spontaneous occurrence and eradication by vitamin C. J Clin Invest 10:209-219

17. Diaz CJ, Mendoza HC, Rodriguez JS 1939 Alkapton, aceton and Kohlehydratmangel. Klin Wochenschr 18:965-969 